\title{
Non-Linear Load Characterisation Using Orthogonal Apparent Power Decompositions
}

\author{
Marko A. Dimitrijevic*, Dejan Stevanovic, Vanco B. Litovski \\ Faculty of Electronics Engineering, University of Nis, \\ Aleksandra Medvedeva 14, 18000 Nis, Serbia \\ marko.dimitrijevic@elfak.ni.ac.rs
}

\begin{abstract}
There is no generally accepted definition for apparent power components in circuits with non-sinusoidal conditions. The decomposition of apparent power has been the subject of many discussions and is still an open topic. Over the years, a number of apparent power decompositions have been proposed and analysed, usually in the context of compensation. These analyses were performed by means of simulations or by calculating quantities using measurements on a simple test circuit. Here, we present a unique system capable of simultaneous apparent power decompositions for nonsinusoidal conditions in real-time. The system can calculate power components related to various power decompositions. The system's capabilities are demonstrated on non-linear load characterization.
\end{abstract}

Index Terms-Reactive power; $\mathrm{Q}$ measurement; Power measurement.

\section{INTRODUCTION}

The dominant cause of non-sinusoidal conditions in a low-voltage electric power distribution grid are electronic appliances, which are non-linear by nature [1]. Electronic devices and appliances are complex systems containing semiconductor devices powered by DC voltage necessary for their polarisation. However, electric power is distributed to end users in the form of an alternating current (AC), which cannot be directly applied to electronic devices. The conversion is performed employing power (AC/DC) converters, which can be modelled as two-port networks with non-linear impedance observed from the power grid side. While keeping the voltage waveform almost unaffected (sinusoidal), they produce current with a seemingly arbitrary waveform causing harmonic distortions. The characterization of the power converter, and thus the electronic appliance containing it, can be performed directly by calculating the harmonics in the current spectrum or indirectly by calculating the quantities that depend on current (and voltage) spectra, such as orthogonal components of apparent power.

The concept of apparent power decomposition is probably one of the most ambiguous notions in electrical engineering. Partly, it comes from the fact that some components are not related to any physical quantities that can be measured directly. When the AC power supply

Manuscript received 9 January, 2020; accepted 30 July, 2020.

This research was partly funded by The Serbian Ministry of Education, Science and Technology Development under Grant No. TR32004 system [2] became dominant [3], some electrical quantities, such as voltage and current root mean square (RMS) values, were introduced with reference to well understood physical quantities in DC circuits. Nevertheless, the product of voltage and current defined in this way is not equal to (active) power in a general case [4]. It was necessary to redefine some basic assumptions and to introduce apparent $(S)$, active $(P)$, and reactive $(Q)$ power. In circuits with pure sinusoidal voltage and current waveforms, these three quantities are related through a quadratic formula $S^{2}=P^{2}+Q^{2}$.

In circuits with non-sinusoidal stimuli or/and with nonlinear loads, it is necessary to take into calculation all the harmonics present in voltage and current spectra. The RMS values, as well as the apparent power, include a contribution from all harmonic components. At this point, it can be concluded, considering purely from the perspective of mathematical combinatorics, that the problem is significantly more complex [5].

There is no generally accepted decomposition of apparent power, nor a unique definition of reactive power in circuits with non-sinusoidal conditions [5], [6]. Over the last eightyfive years, a number of reactive power definitions have been formulated. The decomposition of apparent power has been the subject of many discussions and is still an open topic. At the very beginning of this decades-long debate, $\mathrm{V}$. Karapetoff made the following observation [5]:

"Any definition of power factor that cannot be realized with fairly simple practical measuring instruments will remain a dead letter; on the other hand, a definition that may not be quite rigorous theoretically may prove to be of great practical usefulness if the corresponding measurements are simple and can readily be understood by the average operating engineer."

As A. E. Emanuel noticed few years ago [5], "today's engineers experience better conditions than Karapetoff's generation: available are accurate and versatile instruments capable of measuring any conventional electric quantity defined by the most involving mathematical expressions." Nevertheless, there are no available instruments capable of measuring power components related to more than one decomposition [7]. Quantitative analysis of apparent power components related to various decompositions are presented in the literature [8]-[12]. The presented results were obtained by means of simulation or by calculating quantities 
using measurements on a simple test circuit. These studies have particular interest in the compensation of non-active power. Except for a few studies written by the authors of this manuscript [13], no attempt has been made to characterize non-linear loads using decompositions of apparent power.

Here, a unique system capable of real-time apparent power decompositions for non-sinusoidal conditions will be presented. The system can perform a quantitative analysis of the apparent power components proposed by C. Budeanu [14], IEEE 1459-2010 [15], [16], the standard phase-shift method [17], Kimbark [18], Sharon [19], Fryze [20], Kusters and Moore [21], Shepherd and Zakhikani [22], Dopenbrock [23], and Czarnecki [24], [25]. To demonstrate the system's capabilities, power decompositions were performed and used for the characterization of the number of low-voltage non-linear loads.

The rest of the manuscript is organized as follows. In the second section, the notion of power in circuits with sinusoidal and non-sinusoidal conditions is reviewed. The third section gives details about the widely recognized apparent power decompositions in literature. The fourth section describes a system for real-time power decompositions. The fifth section discusses the results obtained using the described system. Finally, the sixth section concludes the manuscript.

\section{POWER IN CIRCUITS WITH SINUSOIDAL AND NON- SinUSOIDAL CONDITIONS}

In linear circuits [26], a simple sinusoidal voltage source defined in a time domain with the equation

$$
v(t)=\sqrt{2} \times V_{\mathrm{RMS}} \times \sin (\omega t)
$$

yields a sinusoidal current

$$
i(t)=\sqrt{2} \times I_{\mathrm{RMS}} \times \sin (\omega t-\varphi)
$$

In (1) and (2), $V_{R M S}$ and $I_{R M S}$ represent the voltage and current RMS values, respectively. $\omega$ is the fundamental angular frequency, $\varphi$ represents the phase difference between voltage and current waveforms, and $t$ is the time.

The voltage (current) RMS value is equal to

$$
V_{\mathrm{RMS}}=\|v t\|=\sqrt{\frac{1}{T} \int_{0}^{T} v^{2} t d t .}
$$

The instantaneous power is defined as the product of voltage and current

$$
p(t)=v(t) \times i(t)
$$

Active power has a distinct meaning, this is power that can perform some physical work and can be measured as work performed on a load in a given time.

Using (4), the active power can be expressed as the average over a period

$$
P=\frac{1}{T} \int_{t_{0}}^{t_{0}+T} v(t) \times i(t) \times d t
$$

where $t_{0}$ is some arbitrary time after a sinusoidal regime is established and $T$ is the period, $T=2 \pi / \omega$.

Apparent power, $S$, represents the amplitude of the oscillatory component of instantaneous power. It can be expressed as the product of the RMS value of current times and the RMS value of voltage

$$
S=V_{\mathrm{RMS}} \times I_{\mathrm{RMS}}
$$

If the circuit contains non-linear elements, the sinusoidal condition no longer holds and the previous analysis is not applied [27]-[29]. Using a Fourier transform [30], the nonsinusoidal, periodic quantities can be expressed by series:

$$
\begin{gathered}
v(t)=V_{0}+\sum_{k=1}^{\infty} \sqrt{2} \times V_{k} \times \cos \left(k \omega t+\varphi_{\mathrm{V} ; k}\right), \\
i(t)=I_{0}+\sum_{k=1}^{\infty} \sqrt{2} \times I_{k} \times \cos \left(k \omega t+\varphi_{\mathrm{I} ; k}\right) .
\end{gathered}
$$

For the sake of simplicity, the RMS subscripts were dropped; therefore, $V_{k}$ and $I_{k}$ represent RMS values; $\varphi_{\mathrm{V} ; k}$ and $\varphi_{\mathrm{I} ; k}$ are phases of the $k^{\text {th }}$ harmonic of voltage and current. $V_{0}$ and $I_{0}$ denote DC components.

The instantaneous power $p(t)$ calculated using (4) is

$$
\begin{gathered}
p(t)=V_{0} \times I_{0}+ \\
+\sqrt{2} \sum_{k=1}^{\infty}\left(V_{0} I_{k} \times \cos \left(k \omega t+\varphi_{\mathrm{I} ; k}\right)+V_{k} I_{0} \times \cos \left(k \omega t+\varphi_{\mathrm{V} ; k}\right)\right)+ \\
+2 \sum_{k=1}^{\infty} V_{k} I_{k} \times \cos \left(k \omega t+\varphi_{\mathrm{V} ; k}\right) \times \cos \left(k \omega t+\varphi_{\mathrm{I} ; k}\right)+ \\
+2 \sum_{k=1}^{\infty} \sum_{\substack{l=1 \\
l \neq k}}^{\infty} V_{k} I_{l} \times \cos \left(k \omega t+\varphi_{\mathrm{V} ; k}\right) \times \cos \left(l \omega t+\varphi_{\mathrm{I} ; l l}\right) .
\end{gathered}
$$

The first term on the right-hand side of (9) is the DC power. The first sum is the oscillatory component which emerged from the products between DC and harmonic currents and voltages. The terms of the second produce one time-independent value $V_{k} \times I_{k} \times \cos \left(\varphi_{\mathrm{V} ; k}-\varphi_{\mathrm{I} ; k}\right)$ and one oscillatory with an amplitude $V_{k} \times I_{k}$ and angular frequency $2 k \omega$. The third sum represents inter-harmonic products, which can be expanded into two sinusoidal components with frequencies $k+l$ and $|k-l|$ times fundamental frequency, $\omega$.

The active power, defined as the average of instantaneous power over a period, can be calculated from (5) and (9). The integral of the cosine function over the integer number of periods is equal to zero; therefore, integrating (9) over the period gives active power:

$$
P=V_{0} \times I_{0}+\sum_{k=1}^{\infty} V_{k} \times I_{k} \times \cos \left(\varphi_{\mathrm{V} ; k}-\varphi_{\mathrm{I} ; k}\right),
$$




$$
P=P_{0}+P_{1}+P_{\mathrm{H}}
$$

where $P_{0}, P_{1}$, and $P_{H}$ are DC power, active power of the fundamental harmonic, and harmonic active power, respectively.

\section{DECOMPOSITIONS OF APPARENT POWER}

There are a number of apparent power decompositions and proposed relations between apparent power and its components. In this section, the most common ones are presented. The discussion is limited to properties relevant to power component calculation. For more, the reader may consult the references as listed in the sequel. We recommend [5], [6].

\section{A. Budeanu's Apparent Power Decomposition and Definition of Reactive and Distortion Power}

The most known power decomposition is C. Budeanu's decomposition [5], [14], [25]: apparent power consists of three orthogonal components - active, reactive, and distortion power

$$
S=\sqrt{P^{2}+Q_{\mathrm{b}}^{2}+D_{\mathrm{b}}^{2}}
$$

Active power is determined as the average of instantaneous power and can be expressed with (10).

Budeanu's reactive power is postulated using the productto-sum trigonometric transformations applied to each member of the second sum in (9)

$$
Q_{\mathrm{b}}=\sum_{k=1}^{\infty} V_{k} \times I_{k} \times \sin \left(\varphi_{\mathrm{V} ; k}-\varphi_{\mathrm{I} ; k}\right)
$$

The third proposed component is distortion power

$$
\begin{gathered}
D_{\mathrm{b}}^{2}=\sum_{k=1}^{\infty} \sum_{l=k+1}^{\infty}\left(\left(V_{k} \times I_{l}\right)^{2}+\left(V_{l} \times I_{k}\right)^{2}-\right. \\
\left.-2 V_{k} \times V_{l} \times I_{l} \times I_{k} \cos \left(\varphi_{\mathrm{V} ; k}-\varphi_{\mathrm{I} ; k}-\varphi_{\mathrm{V} ; l}+\varphi_{\mathrm{I} ; l}\right)\right) .
\end{gathered}
$$

\section{B. Standard Method for Reactive Power Calculation}

Instruments available on the market and conventional power meters calculate reactive power as the average value of the product of the shifted voltage and current waveforms

$$
Q_{\text {std }}=\frac{1}{T} \int_{t_{0}}^{t_{0}+T} v\left(t-\frac{T}{4}\right) \times i(t) \times d t
$$

This method is widely used due to its simple implementation. The shifted waveform is obtained by a Hilbert transform [17], [31].

Using analysis similar to active power, reactive power can be expressed in the frequency domain as

$$
Q_{\mathrm{std}}=V_{0} \times I_{0}+\sum_{k=1}^{\infty} V_{k} \times I_{k} \times \cos \left(\varphi_{\mathrm{V} ; k}-\varphi_{\mathrm{I} ; k}-\frac{k \times \pi}{2}\right) .
$$

It is obvious that if both waveforms contain a DC component, the reactive power calculated using the phase- shift method will yield an error. The first, $V_{0} \times I_{0}$, term represents DC power and must be eliminated from the sum. This can be easily done by eliminating the DC component in the voltage (or current) spectrum.

\section{IEEE Standard 1459-2010 Definition of Reactive Power}

IEEE Standard 1459-2010 [15], [16] postulates reactive power as a quadratic sum

$$
Q_{\mathrm{IEEE}}=\sqrt{\sum_{k=1}^{\infty} I_{k}^{2} \times V_{k}^{2} \times \sin ^{2}\left(\varphi_{\mathrm{V} ; k}-\varphi_{\mathrm{I} ; k}\right)} .
$$

Equation (17) ensures that the value of the total reactive power $Q_{\text {IEEE }}$ is always equal or bigger than the absolute value of the fundamental component. Nevertheless, the character of the load is undetermined and simple compensation with a single reactive element is not possible.

\section{Kimbark's Power Decomposition}

E. W. Kimbark [18] proposed an apparent power decomposition to two orthogonal components, active and deactive power. Like in Budeanu's decomposition, active power is defined as the average of instantaneous power. Non-active power is further separated into two orthogonal components, Kimbark's reactive and distortion power. Reactive power is calculated by

$$
Q_{\mathrm{k}}=I_{1} \times V_{1} \times \sin \left(\varphi_{\mathrm{V} ; 1}-\varphi_{\mathrm{I} ; 1}\right) .
$$

It includes only a contribution from the first harmonic. The distortion power contains contribution of higher harmonics

$$
D_{\mathrm{k}}=\sqrt{S^{2}-P^{2}-Q_{\mathrm{k}}^{2}}
$$

\section{E. Sharon's Power Decomposition}

This power decomposition [19] includes the already defined active power $P$ and introduces two components: reactive $S_{q}$ and complementary apparent power $S_{c}$

$$
S=\sqrt{P^{2}+S_{\mathrm{q}}^{2}+S_{\mathrm{c}}^{2}}
$$

Reactive apparent power is

$$
S_{\mathrm{q}}=V_{\mathrm{RMS}} \times \sqrt{\sum_{k=1}^{\infty} I_{k}^{2} \sin ^{2}\left(\varphi_{\mathrm{V} ; k}-\varphi_{\mathrm{I} ; k}\right)} .
$$

\section{F. Fryze's Current Decomposition}

Unlike the previously described power decompositions, Fryze's decomposition is postulated in the time domain [20], [25]. This decomposition does not require the calculation of harmonic amplitudes using a Fourier transform. It is based on instantaneous current separation into two quantities, active and reactive current.

Active current is given by

$$
i_{\mathrm{a}}(t)=\frac{P}{V_{\mathrm{RMS}}^{2}} v(t),
$$


while the reactive current is represented with

$$
i_{\mathrm{r}}(t)=i(t)-i_{\mathrm{a}}(t)
$$

The RMS values of active and reactive currents are

$$
I_{\mathrm{a}}=\frac{P}{V_{\mathrm{RMS}}},
$$

and

$$
I_{\mathrm{r}}=\sqrt{I_{\mathrm{RMS}}^{2}-\frac{P^{2}}{V_{\mathrm{RMS}}^{2}}}
$$

Related active and reactive powers are:

$$
\begin{gathered}
P=V_{\mathrm{RMS}} \times I_{\mathrm{a}}, \\
Q_{\mathrm{f}}=V_{\mathrm{RMS}} \times I_{r} .
\end{gathered}
$$

The main advantage of Fryze's decomposition is a simple calculation. In [32], the reactive current is shown as the best choice for load classification.

\section{G. Kusters and Moore's Power Definitions}

Kusters and Moore's decomposition enables reactive power compensation by coupling appropriate capacitances or inductances [21]. Current is represented as the sum of three components: active current, capacitive or inductive reactive currents and residual currents.

Active current is defined in the same way as in Fryze's decomposition, (22). Reactive capacitive and inductive currents are given by

$$
i_{\mathrm{qC}} t=\frac{\dot{v} t}{\|\dot{v} t\|^{2}} \times \frac{1}{\mathrm{~T}} \int_{0}^{\mathrm{T}} \dot{v} t \times i t d t,
$$

and

$$
i_{\mathrm{qL}} t=\frac{\bar{v} t}{\|\bar{v} t\|^{2}} \times \frac{1}{\mathrm{~T}} \int_{0}^{\mathrm{T}} \bar{v} t \times i t d t
$$

where $\dot{v} t=d v / d t$ and $\bar{v} t=\int_{0}^{t} v \tau d \tau$ represent a voltage derivative with respect to time and the integral of voltage over the time, respectively.

This decomposition assumes active, inductive (or capacitive) reactive power, and residual reactive power

$$
S=\sqrt{P^{2}+Q_{\mathrm{L}}^{2}+Q_{\mathrm{Lr}}^{2}}=\sqrt{P^{2}+Q_{\mathrm{C}}^{2}+Q_{\mathrm{Cr}}^{2}},
$$

where capacitive and inductive reactive powers are:

$$
\begin{aligned}
& Q_{\mathrm{C}}=V_{\mathrm{RMS}} \times\left\|i_{\mathrm{qC}} t\right\|, \\
& Q_{\mathrm{L}}=V_{\mathrm{RMS}} \times\left\|i_{\mathrm{qL}} t\right\| .
\end{aligned}
$$

In the frequency domain, capacitive reactive power can be calculated as

$$
Q_{\mathrm{C}}=V_{\mathrm{RMS}} \times \frac{\sum_{k=1}^{\infty} k \times V_{k} \times I_{k} \times \sin \left(\varphi_{\mathrm{V} ; k}-\varphi_{\mathrm{I} ; k}\right)}{\sqrt{\sum_{k=1}^{\infty} k^{2} \times V_{k}^{2}}},
$$

and inductive reactive power as

$$
Q_{\mathrm{L}}=V_{\mathrm{RMS}} \times \frac{\sum_{k=1}^{\infty} \frac{1}{k} \times V_{k} \times I_{k} \times \sin \left(\varphi_{\mathrm{V} ; k}-\varphi_{\mathrm{I} ; k}\right)}{\sqrt{\sum_{k=1}^{\infty} \frac{V_{k}^{2}}{k^{2}}}} .
$$

\section{H. M. Depenbrock's Method}

Depenbrock's method is an improvement on Fryze's method [23]. This method assumes time-domain separation of voltage and current into fundamental $\left(v_{1}, i_{1}, k=1\right.$ in (7)) and non-fundamental $\left(v_{\mathrm{H}}, i_{\mathrm{H}}, k>1\right)$ components.

The equivalent conductance of the fundamental harmonic and the equivalent conductivity of the higher harmonics are defined as:

$$
\begin{gathered}
G=\frac{P}{V_{\mathrm{RMS}}^{2}}, \\
G_{1}=\frac{P_{1}}{V_{1}^{2}}, \\
G_{\mathrm{H}}=\frac{P_{\mathrm{H}}}{V_{\mathrm{RMS}}^{2}-V_{1}^{2}} .
\end{gathered}
$$

Six components of the instantaneous currents are defined as:

$$
\begin{gathered}
i_{1} t=G \times v_{1} t, \\
i_{\mathrm{le}} t=G_{1}-G \times v_{1} t, \\
i_{\mathrm{H}} t=G \times v_{\mathrm{H}} t, \\
i_{\mathrm{He}} t=G_{\mathrm{H}}-G \times v_{\mathrm{H}} t, \\
i_{\mathrm{q}} t=\frac{1}{V_{1}^{2}} \times v_{1}\left(t-\frac{T_{0}}{4}\right) \times \frac{1}{T} \int_{0}^{T} i t \times v_{1}\left(t-\frac{T}{4}\right) d t, \\
i_{\mathrm{d}} t=i t-i_{1} t-i_{\mathrm{le}} t-i_{\mathrm{H}} t-i_{\mathrm{He}} t-i_{\mathrm{q}} t .
\end{gathered}
$$

Based on the defined currents, reactive power $Q$, phasor power $U$, and residual power $N$ are calculated:

$$
\begin{gathered}
Q=V_{\mathrm{RMS}} \times\left\|i_{\mathrm{q}} t\right\|, \\
U=V_{\mathrm{RMS}} \times \sqrt{\left\|i_{\mathrm{le}} t\right\|^{2}+\left\|i_{\mathrm{He}} t\right\|^{2}}, \\
N=V_{\mathrm{RMS}} \times\left\|i_{\mathrm{d}} t\right\| .
\end{gathered}
$$

Therefore, Depenbrock's apparent power decomposition is

$$
S=\sqrt{P^{2}+Q^{2}+U^{2}+N^{2}} .
$$




\section{Shepherd-Zakikhani's Method}

Shepherd-Zakikhani's power decomposition [22] is based on the decomposition of current into three components: active, reactive, and distortion currents:

$$
\begin{gathered}
I_{\mathrm{a}}=\sqrt{\sum_{k \in \mathbb{I} \cap \mathbb{V}} I_{k}^{2} \cos ^{2}\left(\varphi_{\mathrm{V} ; k}-\varphi_{\mathrm{I} ; k}\right)}, \\
I_{\mathrm{r}}=\sqrt{\sum_{k \in \mathbb{I} \cap \mathbb{V}} I_{k}^{2} \sin ^{2}\left(\varphi_{\mathrm{V} ; k}-\varphi_{\mathrm{I} ; k}\right)}, \\
I_{\mathrm{d}}=\sqrt{I^{2}-I_{\mathrm{a}}^{2}-I_{\mathrm{r}}^{2}},
\end{gathered}
$$

whereby the summation is performed only for harmonics that exist simultaneously in the voltage and current spectra.

Apparent power is decomposed into three components, apparent active power

$$
S_{\mathrm{a}}=I_{\mathrm{a}} \times \sqrt{\sum_{k \in \mathbb{I} \cup \mathbb{V}} V_{k}^{2}}
$$

apparent reactive power

$$
S_{\mathrm{r}}=I_{\mathrm{r}} \times \sqrt{\sum_{k \in \mathbb{I} \cup \mathbb{V}} V_{k}^{2}}
$$

and the apparent power of distortion

$$
S_{\mathrm{d}}^{2}=\sum_{k \in \mathbb{I} \cap \mathbb{V}} V_{k}^{2} \times \sum_{k \in \mathbb{I} \mathbb{V}} I_{k}^{2}+\sum_{k \in \mathbb{V} \backslash \mathbb{I}} V_{k}^{2}\left(\sum_{k \in \mathbb{I} \cap \mathbb{V}} I_{k}^{2}+\sum_{k \in \mathbb{I} \backslash \mathbb{V}} I_{k}^{2}\right)
$$

The summation is over index $k$, where $\mathbb{V}$ and $\mathbb{I}$ represent sets of indices corresponding to the order of the harmonics present in the current or voltage spectrum. The square of apparent power equals the sum of squares

$$
S^{2}=S_{\mathrm{a}}^{2}+S_{\mathrm{r}}^{2}+S_{\mathrm{d}}^{2}
$$

\section{J. L. Czarnecki's Method}

Similar to Depenbrock's power decomposition, Czarnecki's power decomposition considers the current components caused by non-sinusoidal voltage power supply or load non-linearity separately [6], [24], [25]. The current RMS value in a circuit can be represented by four orthogonal components: the active current $I_{\mathrm{a}}$, the reactive current $I_{\mathrm{r}}$, the scattering current $I_{\mathrm{s}}$, and the harmonic current $I_{\mathrm{h}}$

$$
I_{\mathrm{RMS}}^{2}=I_{\mathrm{a}}^{2}+I_{\mathrm{r}}^{2}+I_{\mathrm{s}}^{2}+I_{\mathrm{h}}^{2}
$$

The components are given by the following formulas:

$$
\begin{gathered}
I_{\mathrm{a}}=\frac{P}{V_{\mathrm{RMS}}}, \\
I_{\mathrm{r}}=\sqrt{\sum_{k \in \mathbb{V}} B_{k}^{2} \times V_{k}^{2}}, \\
I_{\mathrm{s}}=\sqrt{\sum_{k \in \mathbb{V}} G_{k}-G^{2} \times V_{k}^{2}},
\end{gathered}
$$

$$
I_{\mathrm{h}}=\sqrt{\sum_{k \in \mathbb{I I V}} I_{k}^{2}}
$$

where $\mathbb{V}$ and $\mathbb{I}$ are the sets of voltage and current harmonics' indices. The equivalent conductance of $G$ is calculated identically as in Depenbrock's decomposition, according to expression (35). The equivalent admittance of the $\mathrm{k}^{\text {th }}$ harmonic is

$$
\overline{Y_{k}}=G_{k}+\mathrm{j} B_{k}
$$

Power decomposition is

$$
S^{2}=P^{2}+Q_{\mathrm{r}}^{2}+D_{\mathrm{s}}^{2}+D_{\mathrm{h}}^{2},
$$

whereby reactive power, scattering power, and harmonic power are calculated by the formulas:

$$
\begin{aligned}
& Q_{\mathrm{r}}=V_{\mathrm{RMS}} \times I_{\mathrm{r}}, \\
& D_{\mathrm{s}}=V_{\mathrm{RMS}} \times I_{\mathrm{s}}, \\
& D_{\mathrm{h}}=V_{\mathrm{RMS}} \times I_{\mathrm{h}} .
\end{aligned}
$$

\section{THE System FOR REAL-TIME POWER DECOMPOSITIONS}

Here, we are proposing a unique measurement and data processing system capable of simultaneous apparent power decompositions for non-sinusoidal conditions. The system can perform simultaneous, real-time calculations of power components related to decompositions postulated in the previous section. The simplified system structure is shown in Fig. 1.

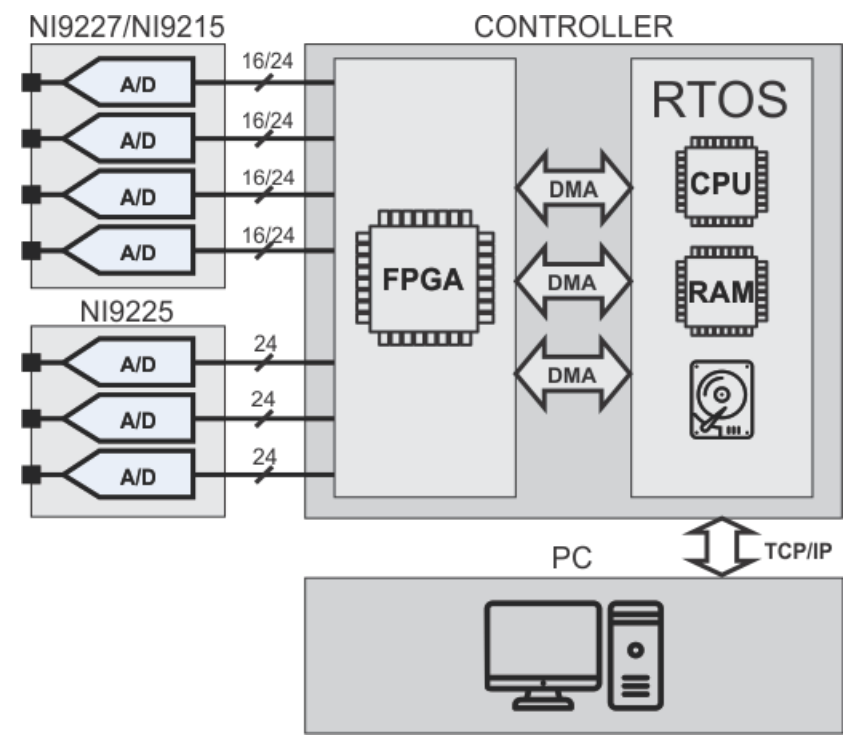

Fig. 1. The simplified system architecture. Acquisition modules are connected to the controller's FPGA. Data transfer between FPGA and CPU is achieved through three 64-bit DMA channels. The system is controlled by a PC, through a TCP/IP network.

The system is prototyped on a virtual instrumentation principle. It consists of an acquisition subsystem, real-time programmable controller with a field programming gate array (FPGA), and system software [33]. The system software is separated into two applications: an application running on a real-time operating system (RTOS) designed 
for data processing, calculations, and data logging and frontend application executing on a general-purpose operating system (GPOS) aimed at data analysis, manipulation, and visualization. A system implemented in this way allows the calculation of a number of power components, as well as other quantities that characterize non-linear loads. This architecture offers many advantages - scalability, openness, and flexibility. The system can easily be extended in functionality - by the number of calculated quantities or in the number of simultaneously measured channels. Flexibility is reflected through the possibility of implementation on various platforms. The system is prototyped using two platforms: a NI-8014 PXI controller equipped with a PXI-7813R card with Xilinx Vitex-II FPGA [34] and cRIO-9024 controller [35].

\section{A. The Acquisition Subsystem}

The acquisition subsystem consists of a connection circuit with current sensors, acquisition modules, and data interface. There are two current ranges available supporting measurements of up to $5 \mathrm{~A}$ and $50 \mathrm{~A}$. The lower current range requires no sensors, and currents smaller than $5 \mathrm{~A}$ can be measured directly using the National Instruments NI9227 acquisition module [36]. NI9227 possesses four channels for simultaneous current sampling with a 24-bit resolution, a 50000 samples per second sampling rate, and a $250 \mathrm{~V}$ RMS channel-to-channel isolation.

A higher current range is achieved with four LEM LA55P Hall effect sensors [37] chosen for good accuracy when measuring the DC current component [38]. However, Hall's effect sensors require a separate power source, which is a disadvantage over the current transformer. Also, DC and temperature calibration, as well as phase compensation, are necessary. The sensor outputs are connected to the National Instruments NI9215 [39] module. The module is equipped with four channels capable of simultaneous voltage sampling with a 16-bit resolution, a $100 \mathrm{kS} / \mathrm{s}$ sampling rate, and a $250 \mathrm{~V}$ RMS channel-to-earth isolation.

Voltages are measured directly using the National Instruments NI9225 module. It has three simultaneously sampling voltage channels with a 24-bit resolution, a 50000 samples per second sampling rate, and a $600 \mathrm{~V}$ RMS channel-to-earth isolation. The $300 \mathrm{~V}$ RMS range is suitable for line-to-neutral measurements of the European power grid (240 V) [6].

The acquisition subsystem is capable of measuring up to the $100^{\text {th }}$ harmonic $(5 \mathrm{kHz})$.

\section{B. Real-Time Controller}

The real-time controller is a data processing element. It has two processing stages: a programmable FPGA stage and a processor-based stage running RTOS [40]. It performs the following tasks:

1. Acquisition control;

2. Parameter calculation;

3. Data logging;

4. Communication with front-end application.

The acquisition control is implemented on FPGA. It is performed in a loop repeating at an interval equal to the integer multiple of FPGA's $40 \mathrm{MHz}$ clock period. The sampling rate is equal to the loop repetition frequency. The sampled data are transferred to the next processing stage via three 64-bit direct memory access (DMA) channels. The acquisition control loop is shown in Fig. 2.

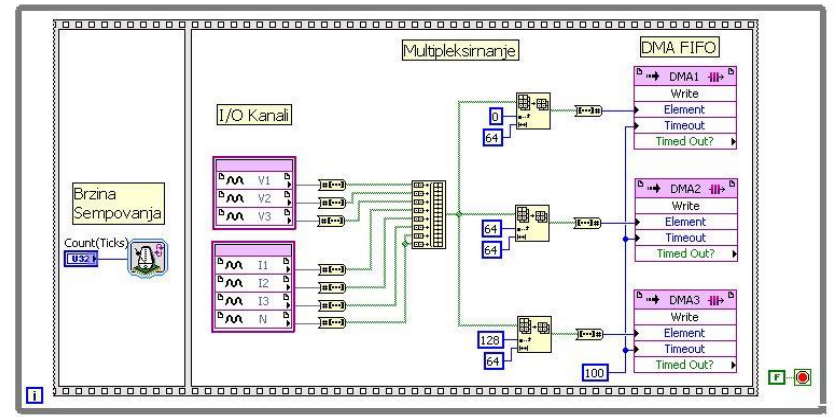

Fig. 2. The acquisition control loop. The sampling rate is set in the first frame. Data acquisition, multiplexing, and transfer are performed in the second frame. This loop is implemented on FPGA.

Parameter calculation, data logging, and communication are part of the RTOS application.

The parameter calculation task implies a Fast Fourier Transform (FFT) [41], numeric integration, and power component calculation. Power decompositions and the calculation of power components using definitions elaborated previously require the calculation of current and voltage harmonics and RMS values. These quantities are obtained from sampled data by means of FFT (voltage and current harmonics) and numerical integration (RMS values, active power).

Active power and RMS values are calculated directly from sampled waveforms using discrete forms of (3) and (5).

Power components are calculated according to specific decomposition using equations given in the previous section (Fig. 3). In practical implementation, infinite sums are substituted with finite sums.

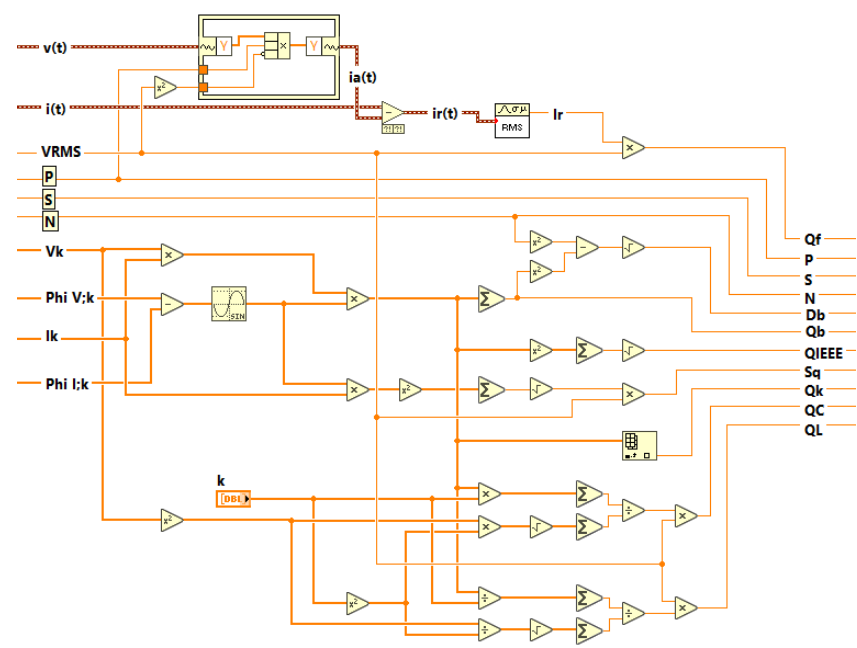

Fig. 3. Part of the code related to power component calculation. The G code follows data flow programming paradigm.

The RTOS application has the capability of saving calculated power components locally on a controller's hard disk or SSD storage. All measured and calculated quantities are available for post-measurement, offline analysis. There are two data saving modes: triggered by user and continuous 
logging with a predefined resolution.

Communication between the RTOS and the front-end application is implemented using a TCP/IP set of protocols.

\section{Front-End Application}

The front-end application serves as a user interface. The application is a virtual instrument implemented using the LabVIEW developing package [42]. It can be executed on any personal computer with installed GPOS, such as Microsoft Windows or Linux. The communication between a PC and real-time controller is established through a TCP/IP network.

The application provides controls over choice of power decompositions and calculated components, data saving modes (user requested and continuous), sampling channels, the highest harmonic taken into calculation and resolution. System configuration - the controller's IP address, name and location of the saved files - is also accessible from the application. Calculated values are presented in numerical form (Fig. 4)

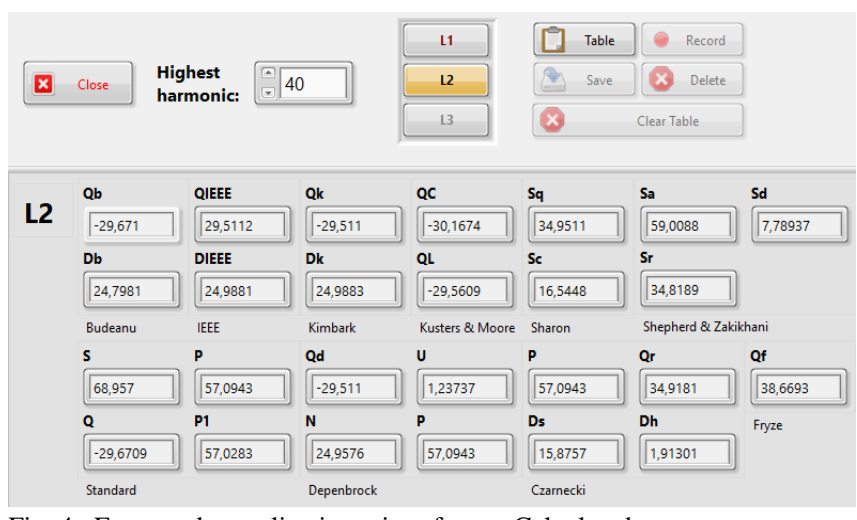

Fig. 4. Front-end application interface. Calculated components are numerically presented.

\section{RESUlTS}

As a demonstration of the system's capabilities, a set of measurements was conducted on loads with constant power consumption and load with variable power consumption.

\section{A. Loads with Constant Power Consumption}

Measurements were performed on indoor compact fluorescent lamps (CFL) with a nominal power of $7 \mathrm{~W}-$ $20 \mathrm{~W}$, light emitting diode lamps (LED) with a nominal power of $3.6 \mathrm{~W}-10 \mathrm{~W}$, and two incandescent lamps $(75 \mathrm{~W}$ and $200 \mathrm{~W}$ ) for reference (Table I). These measurements were performed using user request saving mode. The results are presented in Tables II-V.

Apparent power $(S)$, active power $(P)$, and power components related to Budeanu's $\left(Q_{\mathrm{b}}, D_{\mathrm{b}}\right)$, IEEE 1459$2010\left(Q_{\text {IEEE }}, D_{\text {IEEE }}\right)$ and Kimbark's $\left(Q_{\mathrm{k}}, D_{\mathrm{k}}\right)$ power decompositions are presented in Table II.

Table III shows the power components calculated using Kusters and Moore's $\left(Q_{\mathrm{C}}, Q_{\mathrm{L}}\right)$, Sharon's $\left(S_{\mathrm{q}}, S_{\mathrm{c}}\right)$, and Shepherd and Zakikhani's $\left(S_{\mathrm{a}}, S_{\mathrm{r}}, S_{\mathrm{d}}\right.$ ) decompositions.

The following values are displayed in Table IV: reactive power $\left(Q_{\mathrm{d}}\right)$, residual power $(N)$, and phasor power $(U)$ defined according to Depenbrock's decomposition; active power ( $P$ ); scattering power $\left(D_{\mathrm{s}}\right)$, reactive power $\left(Q_{\mathrm{r}}\right)$, and harmonic power $\left(D_{\mathrm{h}}\right)$ postulated by Czarnecki.

Table V contains values of reactive power calculated using a standard, phase-shift method ( $Q_{\text {std }}$ ), Fryze's reactive power $\left(Q_{\mathrm{f}}\right)$, and fundamental active power $\left(P_{1}\right)$.

A comparison of active, reactive, and distortion power defined using Budeanu's, IEEE, and Kimbark's decompositions are in agreement with well-known fact that LED and CFL lamps are non-linear loads $\left(D_{\mathrm{b}}>Q_{\mathrm{b}}\right.$, $\left.D_{\mathrm{b}}>P\right)$. Absolute values of related reactive powers $\left(Q_{\mathrm{b}}\right.$, $Q_{\text {IEEE }}, Q_{\mathrm{k}}$ ) are almost equal, suggesting that the contributions of a higher harmonic to reactive power are negligible. Furthermore, the contribution of fundamental active power $\left(P_{1}\right)$ to total active power $(P)$ is dominant for all examined loads.

Distortion power has a non-zero value even for pure linear loads, such as incandescent lamps due to distorted line voltage. During the conducted measurements, the voltage is distorted with $T H D_{\mathrm{v}}<3 \%$.

Reactive power calculated from Fryze's definition is equal to non-active power, $Q_{\mathrm{f}}=\sqrt{S^{2}-P^{2}}$.

The standard method, implemented in many instruments and power meters, yields values unequal to any reactive power definition.

The components of power decompositions can be used for load recognition and classification. The non-intrusive load monitoring (NILM) method using those values as signatures is presented in [43].

TABLE I. CONSTANT POWER CONSUMPTION LOADS.

\begin{tabular}{|c|c|c|c|}
\hline No & Load & No & Load \\
\hline 1 & Incandescent 75 W & 11 & CFL 7 W spot \\
\hline 2 & Incandescent 200 W & 12 & CFL 9 W bulb \\
\hline 3 & CFL 20W tube 1 & 13 & LED 6 W bulb 470 lm \\
\hline 4 & CFL 20W helix 1 & 14 & LED 7 W bulb 400 lm \\
\hline 5 & CFL 20 W helix 2 & 15 & LED 3.6 W bulb 250 lm \\
\hline 6 & CFL 20 W tube 2 & 16 & CFL 7 W bulb \\
\hline 7 & CFL 11 W tube 1 & 17 & LED 10 W bulb \\
\hline 8 & CFL 11 W tube 2 & 18 & LED 9 W bulb 806 lm \\
\hline 9 & CFL 11 W tube 3 & 19 & LED 10 W bulb 700 lm \\
\hline 10 & CFL 20 W bulb 1 & 20 & C \\
\hline
\end{tabular}


TABLE II. APPARENT POWER (S) [VA], ACTIVE POWER (P) [W], BUDEANU, IEEE, AND KIMBARK POWER DECOMPOSITIONS [VAR].

\begin{tabular}{|c|c|c|c|c|c|c|c|c|}
\hline \multirow{2}{*}{ No } & \multirow{2}{*}{$\boldsymbol{S}$} & $\boldsymbol{P}$ & \multicolumn{2}{|c|}{ Budeanu } & \multicolumn{2}{|c|}{ IEEE } & \multicolumn{2}{c|}{ Kimbark } \\
\cline { 3 - 8 } & & $\boldsymbol{Q}_{\mathbf{b}}$ & $\boldsymbol{D}_{\mathbf{b}}$ & $\boldsymbol{Q}$ & $\boldsymbol{D}$ & $\boldsymbol{Q}_{\mathbf{k}}$ & $\boldsymbol{D}_{\mathbf{k}}$ \\
\hline 1 & 73.74 & 71.30 & 0.84 & 18.80 & 0.84 & 18.80 & 0.84 & 18.80 \\
\hline 2 & 190.02 & 189.05 & 2.11 & 19.08 & 2.10 & 19.08 & 2.10 & 19.08 \\
\hline 3 & 35.57 & 17.35 & -8.89 & 29.76 & 8.94 & 29.74 & -8.94 & 29.74 \\
\hline 4 & 35.81 & 17.89 & -9.21 & 29.62 & 9.27 & 29.61 & -9.26 & 29.61 \\
\hline 5 & 35.87 & 17.83 & -9.19 & 29.74 & 9.24 & 29.72 & -9.24 & 29.72 \\
\hline 6 & 35.45 & 18.36 & -8.63 & 29.07 & 8.71 & 29.05 & -8.71 & 29.05 \\
\hline 7 & 24.54 & 9.76 & -5.76 & 21.76 & 5.77 & 21.76 & -5.77 & 21.76 \\
\hline 8 & 28.72 & 12.86 & -6.74 & 24.77 & 6.80 & 24.76 & -6.80 & 24.76 \\
\hline 9 & 25.00 & 9.77 & -5.34 & 22.38 & 5.38 & 22.38 & -5.38 & 22.38 \\
\hline 10 & 33.70 & 17.25 & -8.38 & 27.70 & 8.41 & 27.69 & -8.41 & 27.69 \\
\hline 11 & 20.52 & 5.91 & -2.99 & 19.42 & 3.00 & 19.42 & -3.00 & 19.42 \\
\hline 12 & 24.62 & 9.83 & -4.77 & 22.06 & 4.81 & 22.06 & -4.81 & 22.06 \\
\hline 13 & 21.28 & 6.43 & -2.77 & 20.09 & 2.82 & 20.09 & -2.82 & 20.09 \\
\hline 14 & 19.87 & 5.98 & -4.01 & 18.52 & 3.99 & 18.52 & -3.99 & 18.52 \\
\hline 15 & 19.48 & 6.08 & -2.48 & 18.34 & 2.50 & 18.34 & -2.50 & 18.34 \\
\hline 16 & 19.47 & 3.27 & -4.85 & 18.57 & 4.85 & 18.57 & -4.85 & 18.57 \\
\hline 17 & 20.68 & 5.69 & -2.55 & 19.72 & 2.58 & 19.71 & -2.58 & 19.71 \\
\hline 18 & 21.76 & 11.48 & -2.71 & 18.29 & 2.70 & 18.29 & -2.70 & 18.29 \\
\hline 19 & 24.29 & 9.24 & -3.19 & 22.24 & 3.31 & 22.22 & -3.31 & 22.22 \\
\hline 20 & 21.18 & 9.60 & -2.76 & 18.67 & 2.80 & 18.67 & -2.80 & 18.67 \\
\hline
\end{tabular}

TABLE III. KUSTERS \& MOORE, SHARON, AND SHEPHERD \& ZAKIKHANI POWER DECOMPOSITIONS [VAR].

\begin{tabular}{|c|c|c|c|c|c|c|c|}
\hline \multirow{2}{*}{ No } & \multicolumn{2}{|c|}{ Kusters \& Moore } & \multicolumn{2}{|c|}{ Sharon } & \multicolumn{3}{c|}{ Shepherd \& Zakikhani } \\
\cline { 2 - 8 } & $\boldsymbol{Q}_{\mathbf{C}}$ & $\boldsymbol{Q}_{\mathbf{L}}$ & $\boldsymbol{S}_{\mathbf{q}}$ & $\boldsymbol{S}_{\mathbf{c}}$ & $\boldsymbol{S}_{\mathbf{a}}$ & $\boldsymbol{S}_{\mathbf{r}}$ & $\boldsymbol{S}_{\mathbf{d}}$ \\
\hline 1 & 0.86 & 0.84 & 0.90 & 18.79 & 71.32 & 18.75 & 0.89 \\
\hline 2 & 2.14 & 2.11 & 2.16 & 19.07 & 189.05 & 19.06 & 2.16 \\
\hline 3 & -8.83 & -8.93 & 15.76 & 26.76 & 23.90 & 23.22 & 12.45 \\
\hline 4 & -9.16 & -9.26 & 15.94 & 26.61 & 24.46 & 22.77 & 12.86 \\
\hline 5 & -9.14 & -9.23 & 15.84 & 26.80 & 24.39 & 22.88 & 12.97 \\
\hline 6 & -8.50 & -8.70 & 15.33 & 26.17 & 25.07 & 21.93 & 12.16 \\
\hline 7 & -5.81 & -5.77 & 9.82 & 20.26 & 13.36 & 18.97 & 8.00 \\
\hline 8 & -6.63 & -6.79 & 11.77 & 22.82 & 17.97 & 20.28 & 9.50 \\
\hline 9 & -5.28 & -5.37 & 9.32 & 21.04 & 13.59 & 19.71 & 7.23 \\
\hline 10 & -8.37 & -8.41 & 14.52 & 25.03 & 23.92 & 20.71 & 11.58 \\
\hline 11 & -2.99 & -2.99 & 5.26 & 18.93 & 8.01 & 18.36 & 4.43 \\
\hline 12 & -4.72 & -4.80 & 8.76 & 20.80 & 13.46 & 19.30 & 7.23 \\
\hline 13 & -2.63 & -2.81 & 4.97 & 19.66 & 9.15 & 18.75 & 4.19 \\
\hline 14 & -4.10 & -4.00 & 5.11 & 18.24 & 6.10 & 18.22 & 5.07 \\
\hline 15 & -2.38 & -2.50 & 3.30 & 18.21 & 6.54 & 18.13 & 2.79 \\
\hline 16 & -4.87 & -4.85 & 5.77 & 18.31 & 3.63 & 18.26 & 5.70 \\
\hline 17 & -2.48 & -2.57 & 4.69 & 19.32 & 7.96 & 18.69 & 3.86 \\
\hline 18 & -2.75 & -2.70 & 2.78 & 18.28 & 11.48 & 18.28 & 2.76 \\
\hline 19 & -2.74 & -3.29 & 7.70 & 21.10 & 13.09 & 19.48 & 6.25 \\
\hline 20 & -2.55 & -2.80 & 4.48 & 18.34 & 10.26 & 18.17 & 3.64 \\
\hline
\end{tabular}

TABLE IV. ACTIVE POWER [W], DEPENBROCK, AND CZARNECKI POWER DECOMPOSITIONS [VAR].

\begin{tabular}{|c|c|c|c|c|c|c|c|}
\hline \multirow[b]{2}{*}{ No } & \multicolumn{3}{|c|}{ Depenbrock } & \multirow[b]{2}{*}{$P$} & \multicolumn{3}{|c|}{ Czarnecki } \\
\hline & $Q_{\mathrm{d}}$ & $N$ & $U$ & & $D_{\mathrm{s}}$ & $Q_{\mathrm{r}}$ & $D_{\mathrm{h}}$ \\
\hline 1 & 0.84 & 18.73 & 1.63 & 71.30 & 0.47 & 0.90 & 0.13 \\
\hline 2 & 2.10 & 18.96 & 2.18 & 189.05 & 0.86 & 2.16 & 0.18 \\
\hline 3 & -8.94 & 29.21 & 5.60 & 17.35 & 17.24 & 14.75 & 7.98 \\
\hline 4 & -9.27 & 29.37 & 3.69 & 17.89 & 17.50 & 15.23 & 6.71 \\
\hline 5 & -9.25 & 29.35 & 4.71 & 17.83 & 17.66 & 15.24 & 6.62 \\
\hline 6 & -8.72 & 28.79 & 3.90 & 18.36 & 17.92 & 12.97 & 10.32 \\
\hline 7 & -5.77 & 21.65 & 2.19 & 9.76 & 9.50 & 9.32 & 4.19 \\
\hline 8 & -6.80 & 24.31 & 4.68 & 12.86 & 13.36 & 11.29 & 5.99 \\
\hline 9 & -5.38 & 22.21 & 2.69 & 9.77 & 9.56 & 7.78 & 6.62 \\
\hline 10 & -8.42 & 27.33 & 4.46 & 17.25 & 17.00 & 12.52 & 8.32 \\
\hline 11 & -3.00 & 19.19 & 2.96 & 5.91 & 5.56 & 5.09 & 1.75 \\
\hline 12 & -4.81 & 22.01 & 1.42 & 9.83 & 9.44 & 7.80 & 4.95 \\
\hline 13 & -2.82 & 20.01 & 1.77 & 6.43 & 7.26 & 4.83 & 1.76 \\
\hline 14 & -3.99 & 18.47 & 1.37 & 5.98 & 1.24 & 5.10 & 0.68 \\
\hline 15 & -2.51 & 18.32 & 0.62 & 6.08 & 2.68 & 3.10 & 2.17 \\
\hline 16 & -4.85 & 18.54 & 1.05 & 3.27 & 1.64 & 5.75 & 0.95 \\
\hline 17 & -2.58 & 19.68 & 1.11 & 5.69 & 5.73 & 4.58 & 2.56 \\
\hline 18 & -2.70 & 18.29 & 0.03 & 11.48 & 0.21 & 2.77 & 0.32 \\
\hline 19 & -3.31 & 22.19 & 1.16 & 9.24 & 10.71 & 6.85 & 4.44 \\
\hline 20 & -2.80 & 18.60 & 1.57 & 9.60 & 3.95 & 4.16 & 3.34 \\
\hline
\end{tabular}


TABLE V. FUNDAMENTAL HARMONIC POWER [W], STANDARD, AND FRYZE'S REACTIVE POWER [VAR].

\begin{tabular}{|c|c|c|c|c|c|c|c|}
\hline No & $\boldsymbol{Q}_{\text {std }}$ & $\boldsymbol{Q}_{\mathrm{f}}$ & $\boldsymbol{P}_{\mathbf{1}}$ & $\mathbf{N o}$ & $\boldsymbol{Q}_{\text {std }}$ & $\boldsymbol{Q}_{\mathrm{f}}$ & $\boldsymbol{P}_{\mathbf{1}}$ \\
\hline 1 & 0.83 & 18.81 & 71.27 & 11 & -2.99 & 19.65 & 5.87 \\
\hline 2 & 1.98 & 19.20 & 188.93 & 12 & -4.80 & 22.57 & 9.75 \\
\hline 3 & -8.87 & 31.05 & 17.20 & 13 & -2.76 & 20.28 & 6.36 \\
\hline 4 & -9.22 & 31.02 & 17.73 & 14 & -4.01 & 18.95 & 6.03 \\
\hline 5 & -9.17 & 31.13 & 17.66 & 15 & -2.46 & 18.51 & 6.09 \\
\hline 6 & -8.68 & 30.33 & 18.17 & 16 & -4.87 & 19.19 & 3.30 \\
\hline 7 & -5.77 & 22.51 & 9.66 & 17 & -2.55 & 19.88 & 5.64 \\
\hline 8 & -6.74 & 25.68 & 12.71 & 18 & -2.75 & 18.49 & 11.47 \\
\hline 9 & -5.34 & 23.01 & 9.68 & 19 & -3.19 & 22.46 & 9.18 \\
\hline 10 & -8.38 & 28.94 & 17.09 & 20 & -2.78 & 18.88 & 9.64 \\
\hline
\end{tabular}

\section{B. Load with Variable Power Consumption}

A typical representative of this group is a personal computer [44]. The power consumption depends on many parameters - processor (both CPU and GPU) utilization, storage device activity, peripherals, the battery, etc. We used a laptop computer with Intel Core 7i CPU, Nvidia GPU, 16 GB RAM, and 1 TB SSD + 1 TB HDD storage. During the test, the battery was fully charged.

The apparent power, active power, and other power components were captured in a one-hour interval, with a $0.5 \mathrm{~s}$ resolution (values are averaged for 25 periods). CPU utilization was increased from $10 \%$ to $60 \%$ in 10 percent increments at 10 -minute intervals.

The simplest power decomposition involving apparent, active, and nonactive (Fryze's reactive) power is shown in Fig. 5.

A steady increase of active and apparent power related to CPU utilization can be observed. Power transients are the consequences of the processes that are performed in the background. They are stochastic in nature.

The power components defined by some apparent power decompositions are shown in Figs. 6.-10.

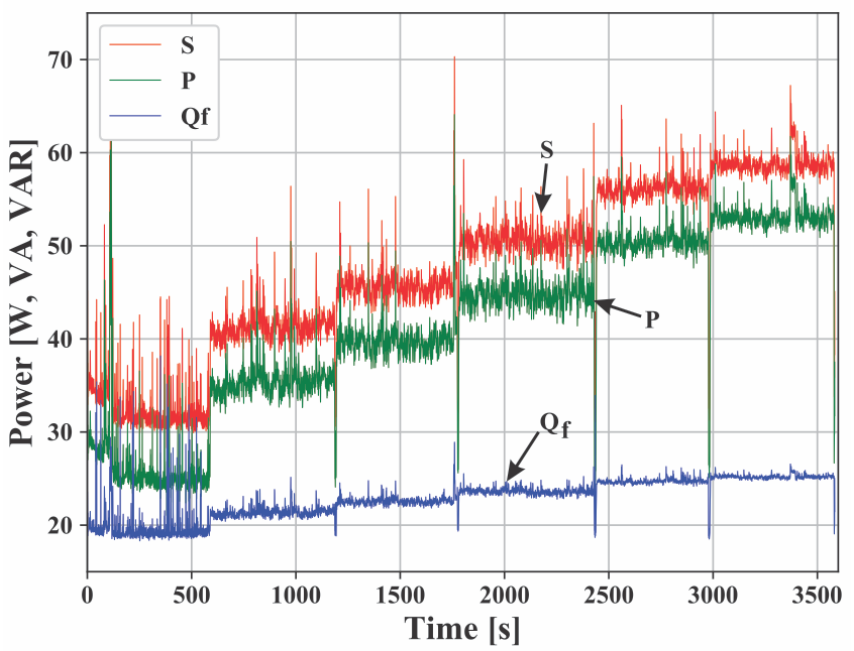

Fig. 5. Apparent power $S$, active $P$, and Fryze's reactive power $Q_{\mathrm{f}}$. Equations (26) and (27).

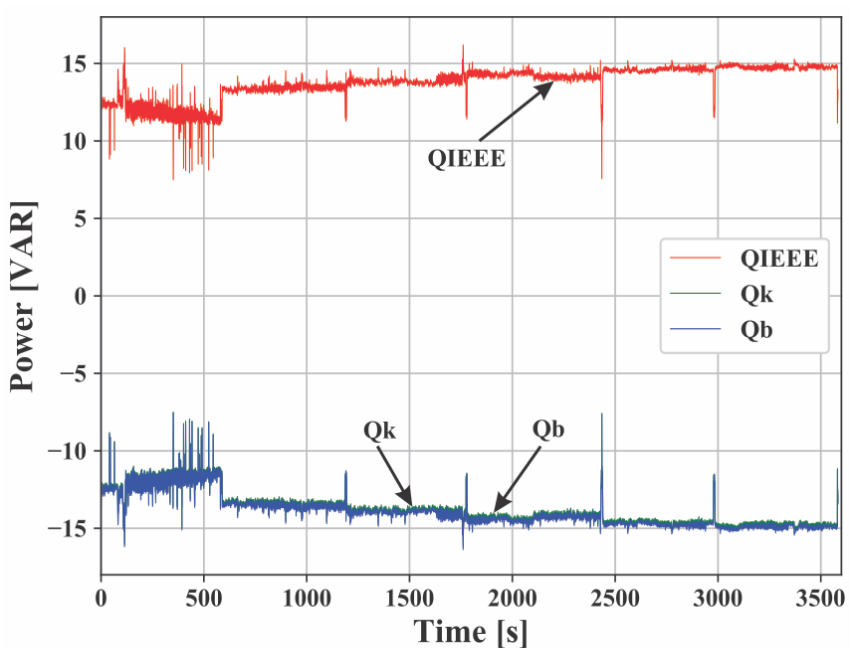

Fig. 6. IEEE 1459-2010 ( $\left.Q_{\mathrm{IEEE}}\right)$, Kimbark's ( $\left.Q_{\mathrm{k}}\right)$, and Budeanu's reactive power $\left(Q_{\mathrm{b}}\right)$. The values of the last two are almost equal, suggesting that the contributions of the higher harmonic to reactive power are negligible. Equations (13), (17), and (18). 


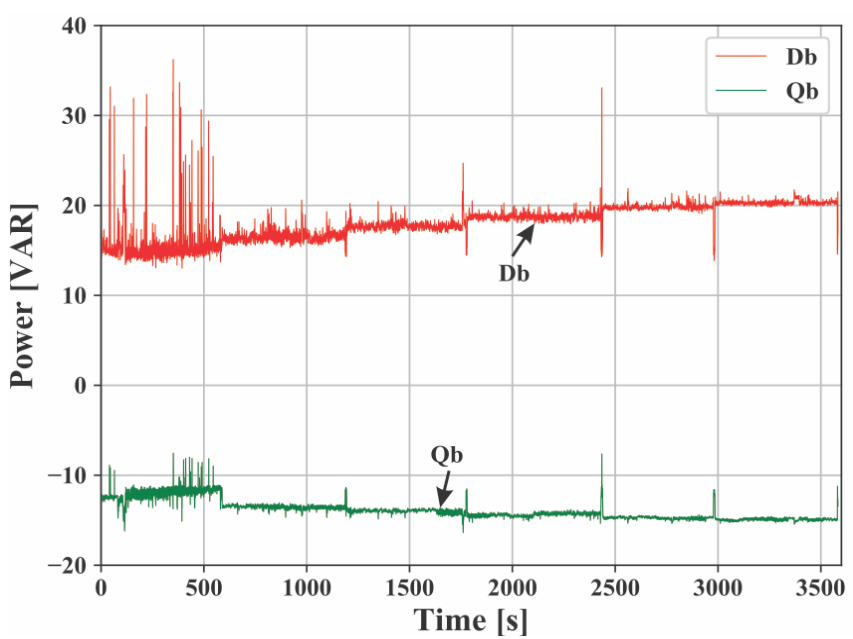

Fig. 7. Budeanu's decomposition, reactive $Q_{\mathrm{b}}$, and distortion power $D_{\mathrm{b}}$. Equations (13) and (14).

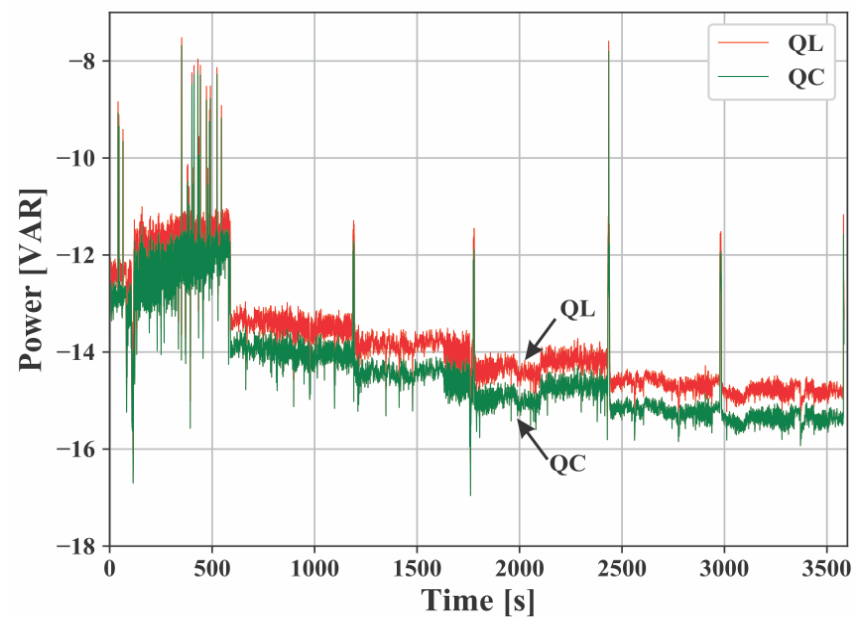

Fig. 8. Kusters and Moore's inductive $Q_{\mathrm{L}}$ and capacitive reactive $Q_{\mathrm{C}}$ powers according to (33) and (34).

\section{CONCLUSIONS}

Apparent power decompositions and alternative definitions of reactive power have been the subject of many debates. These discussions were conducted with particular interest in reactive power compensation. At the same time, a significant increase in the number of non-linear devices has had a tremendous impact on the power grid, causing the necessity to characterize them.

In this manuscript, we have presented a prototype of a measurement and data processing system for real-time, simultaneous apparent power decompositions. The system can perform the calculation of power components related to virtually all power decompositions presented in the literature. The system's functionality is demonstrated on constant and variable power consumption load characterization.

\section{ACKNOWLEDGMENT}

Some graphic elements used in this manuscript, illustrations (Fig. 1.), have been downloaded from: https://www.flaticon.com/authors/nikita-golubev.

Processor utilization was accomplished using CPUGrabEx application: https://www.the-

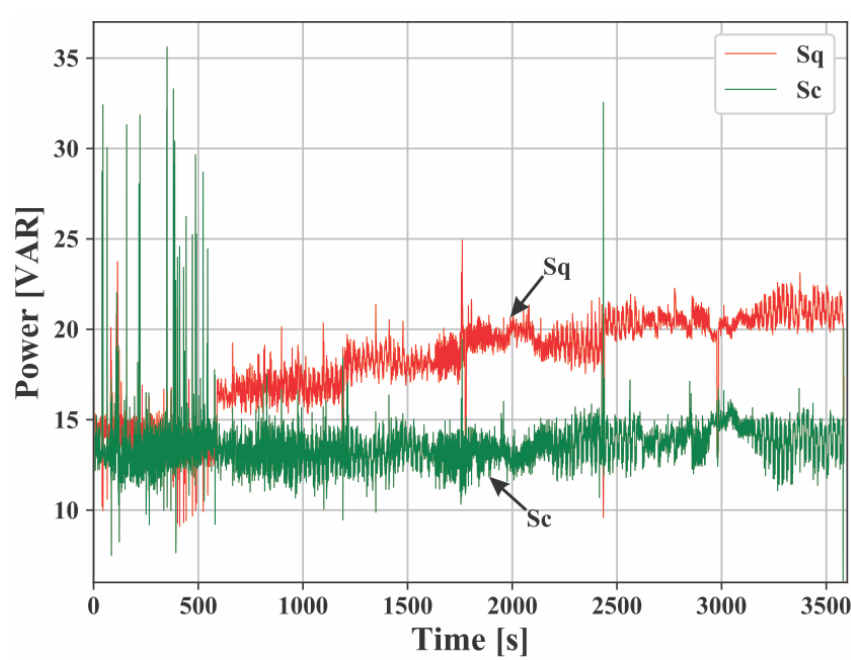

Fig. 9. Sharon's apparent power decomposition, (21).

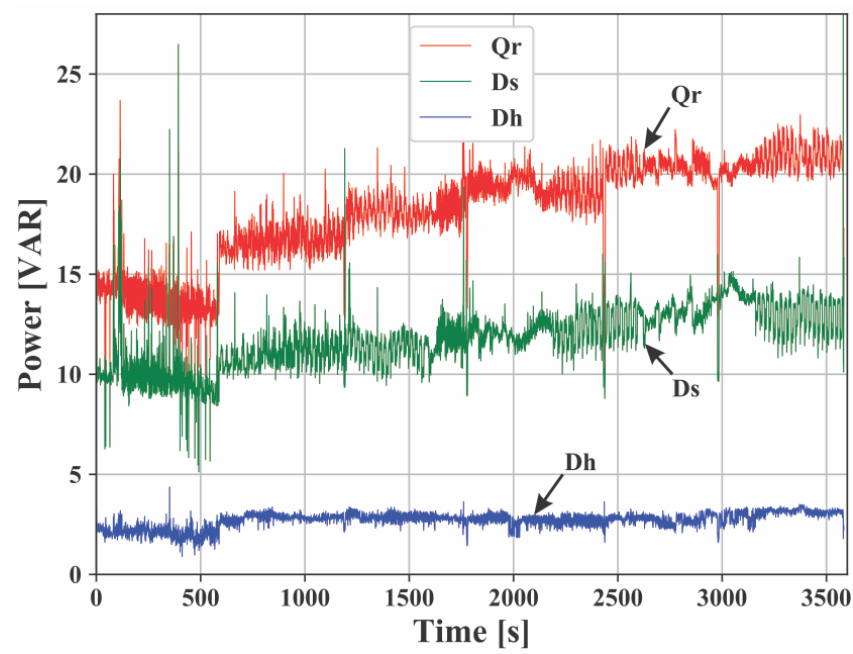

Fig. 10. Czarnecki's apparent power decomposition, (62)-(64).

sz.com/products/cpugrabex/.

\section{CONFLICTS OF INTEREST}

The authors declare that they have no conflicts of interest.

\section{REFERENCES}

[1] G. J. Wakileh, "Harmonics in power systems - Their Causes", in Power Systems Harmonics - Fundaments, Analysis and Filter Design. Springer, Berlin, Heidelberg, 2001, pp. 45-80. DOI: 10.1007/978-3662-04343-1 4.

[2] N. Tesla, "System of electrical power transmission", U.S. Patent No. 511,560, 26 Dec., 1893.

[3] T. McNichol, AC/DC: The Savage Tale of the First Standards War. John Wiley and Sons, 2011.

[4] W. H. Beaty and G. D. Fink, "Alternating-current generators", in Standard Handbook for Electrical Engineers. New York: McGrawHill Education, 2013.

[5] A. E. Emanuel, "Apparent power resolution for nonsinusoidal singlephase systems", in Power Definitions and the Physical Mechanism of Power Flow. John Wiley and Sons, 2010, pp. 93-138. DOI: 10.1002/9780470667149.

[6] L. S. Czarnecki, "Harmonics and power phenomena", in Wiley Encyclopedia of Electrical and Electronics Engineering, J. G. Webster, Ed. John Wiley \& Sons, 1999. DOI: 10.1002/047134608x.W3711.

[7] D. Gallo, C. Landi, and M. Luiso, "Power meter verification issue: Reactive power measurement in non sinusoidal conditions", in Proc. of 2015 IEEE International Instrumentation and Measurement Technology Conference (I2MTC), 2015, pp. 1255-1260. DOI 10.1109/I2MTC.2015.7151453. 
[8] M. E. Balci and M. H. Hocaoglu, "Quantitative comparison of power decompositions", Electric Power Systems Research, vol. 78, no. 3, p. 12, 2008. DOI: 10.1016/j.epsr.2007.02.010.

[9] R. de A. Coelho et al., "Comparative analysis of power definitions in nonsinusoidal conditions - A case study", in Proc. of 2018 Simposio Brasileiro de Sistemas Eletricos (SBSE), 2018, pp. 1-6. DOI: 10.1109/SBSE.2018.8395571.

[10] P. Janik and Z. Wacławek, "Estimation of power components for nonsinusoidal currents and voltages regarded as power quality indices", in Proc. of International Conference on Renewable Energies and Power Quality (ICREPQ'12), 2012, pp. 1225-1229. DOI: 10.24084/repqj10.654.

[11] A. Mansour, Z. Chengning, and H. Nasry, "Measurement of power components in balanced and unbalanced three-phase systems under nonsinusoidal operating conditions by using IEEE standard 14592010 and Fourier analysis", in Proc. of 2013 The International Conference on Technological Advances in Electrical, Electronics and Computer Engineering (TAEECE), 2013, pp. 166-171. DOI: 10.1109/TAEECE.2013.6557216

[12] S. Pajic and A. E. Emanuel, "Modern apparent power definitions: Theoretical versus practical Approach-the general case", IEEE Transactions on Power Delivery, vol. 21, no. 4, pp. 1787-1792, 2006. DOI: 10.1109/TPWRD.2006.876647.

[13] M. Dimitrijević and V. Litovski, "Quantitative analysis of reactive power definitions for small non-linear loads", in Proc. of Small System Simulation Simposium 2012, Niš, 2012, pp. 158-164.

[14] C. Budeanu, Puissances réactives et fictives. Inst. National Roumain pour l'Etude de l'Aménagement et de l'Utilisation des Sources d'Énergie, 1927, p. 360.

[15] IEEE Standard 1459-2010 Definitions for the Measurement of Electric Power Quantities under Sinusoidal, Nonsinusoidal, Balanced, or Unbalanced Conditions (revision), IEEE Std. 14592010, IEEE Power and Energy Society, 2010.

[16] E. So, "IEEE trial-use Standard 1459-2000 definitions for the measurement of electric power quantities under sinusoidal, nonsinusoidal, balanced, or unbalanced conditions", in Proc. of 2002 IEEE Power Engineering Society Winter Meeting, 2002, vol. 2, pp 1421-1424. DOI: 10.1109/PESW.2002.985249.

[17] M. Saitou and T. Shimizu, "Generalized theory of instantaneous active and reactive powers in single-phase circuits based on Hilbert transform", in Proc. of 2002 IEEE 33rd Annual IEEE Power Electronics Specialists Conference, 2002, vol. 3, pp. 1419-1424. DOI: 10.1109/PSEC.2002.1022375.

[18] E. W. Kimbark, Power System Stability. Wiley-IEEE Press, 1995. DOI: $10.1109 / 9780470545614$

[19] D. Sharon, "Reactive-power definitions and power-factor improvement in nonlinear systems", Proceedings of the Institution of Electrical Engineers, vol. 120, no. 6, pp. 704-706, 1973. DOI: 10.1049/piee.1973.0155.

[20] S. Fryze, "Wirk-, blind-, scheinleistung- und elektrischen stromkreisen mit nichtsinusoidalformingem verlauf von strom und spannung", Elektrotechnishe Zeitschrift, no. 25, pp. 596-599, 1932.

[21] N. L. Kusters and W. J. M. Moore, "On the definition of reactive power under nonsinusoidal conditions", IEEE Trans. Power Apparatus Systems, vol. 5, no. 99, pp. 1845-1854, 1980. DOI: 10.1109/TPAS.1980.319833.

[22] W. Shepherd and P. Zakikhani, "Suggested definition of reactive power for nonsinusoidal systems", Proceedings of the Institution of Electrical Engineers, vol. 119, no. 9, pp. 1361-1362, 1972. DOI: 10.1049/piee.1972.0268.

[23] M. Depenbrock, "The FBD-method, a generally applicable tool for analyzing power relations", IEEE Transactions on Power Systems, vol. 8, no. 2, pp. 381-387, 1993. DOI: 10.1109/59.260849.

[24] L. S. Czarnecki, "Powers in nonsinusoidal networks: Their interpretation, analysis and measurement", IEEE Trans. Instrumental Measurements, vol. 39, no. 2, p. 5, Apr. 1990. DOI: $10.1109 / 19.52512$.

[25] L. S. Czarnecki, "Budeanu and Fryze: Two frameworks for interpreting power properties of circuits with nonsinusoidal voltages and currents", Electrical Engineering, vol. 80, no. 6, pp. 359-367, 1997. DOI: 10.1007/BF01232925.

[26] N. M. Tabatabaei, A. J. Aghbolaghi, N. Bizon, and F. Blaabjerg, "Fundamentals of reactive power in AC power systems", in Reactive Power Control in AC Power Systems: Fundamentals and Current Issues. Springer International Publishing, 2017, pp. 49-116. DOI: 10.1007/978-3-319-51118-4_2.

[27] A. A. Eisa, M. M. A. Aziz, and H. K. M. Youssef, "New notions suggested to power theory development Part 1: Analytical derivation", in Proc. of 2008 IEEE Power and Energy Society General Meeting Conversion and Delivery of Electrical Energy in the 21st Century, 2008, pp. 1-7. DOI: 10.1109/PES.2008.4596415.

[28] A. A. Eisa, M. M. A. Aziz, and H. K. M. Youssef, "New notions suggested to power theory development Part 2: A concept for sharing the responsibility of harmonics", in Proc. of 2008 IEEE Power and Energy Society General Meeting - Conversion and Delivery of Electrical Energy in the 21st Century, 2008, pp. 1-4. DOI: 10.1109/PES.2008.4596534.

[29] N. M. Tabatabaei, A. J. Aghbolaghi, N. Bizon, and F. Blaabjerg, "Reactive power compensation in energy transmission systems with sinusoidal and nonsinusoidal currents", in Reactive Power Control in AC Power Systems: Fundamentals and Current Issues. Springer International Publishing, 2017, pp. 137-190. DOI: 10.1007/978-3319-51118-4_4.

[30] G. B. Folland, "Fourier series", in Fourier Analysis and Its Applications. American Mathematical Society, 2009, pp. 18-61.

[31] P. Hao, W. Zanji, and C. Jianye, "A measuring method of the singlephase AC frequency, phase, and reactive power based on the Hilbert filtering", IEEE Transactions on Instrumentation and Measurement, vol. 56, no. 3, pp. 918-923, 2007. DOI: 10.1109/TIM.2007.894885.

[32] T. D. Huang, W. Wang, and K. Lian, "A new power signature for nonintrusive appliance load monitoring”, IEEE Transactions on Smart Grid, vol. 6, no. 4, pp. 1994-1995, 2015. DOI 10.1109/TSG.2015.2415456.

[33] M. Dimitrijević, "Elektronski sistem za analizu polifaznih opterećenja baziran na FPGA", Ph.D dissertation, Faculty of Electronic Engineering, University of Niš, Niš, 2012.

[34] NI PXI-7813R Reference, NI, 2019. [Online]. Available: http://zone.ni.com/reference/en-XX/help/371508V01/target2devicehelp/ni_pxi-7813r/

[35] NI cRIO-9024 User Manual and Specifications, NI, 2015. [Online]. Available: http://www.ni.com/pdf/manuals/375233f.pdf

[36] NI 9227 Operating Instructions and Specifications, NI, 2014. [Online]. Available: http://www.ni.com/pdf/manuals/374707e.pdf

[37] Current Transducer LA 55-P, LEM, 2018. [Online]. Available: https://www.lem.com/sites/default/files/products_datasheets/la_55p_e.pdf

[38] J. G. Webster, "Current Measurement", in The Measurement, Instrumentation, and Sensors: Handbook. CRC Press, 1999, pp. 1200-1217.

[39] NI 9215 Operating Instructions and Specifications, NI, 2007. [Online]. Available: http://www.automationlabs.ru/images/hardware/crio9215.pdf

[40] C. Jarvis, K. Kinsella, and P. Timpanaro, "Phar Lap ETS"M - An industrial-strength RTOS white paper", VenturCom, 2001. [Online]. Available: https://www.yumpu.com/en/document/read/6175920/pharlap-ets-aaa-an-industrial-strength-rtos-infoteam-software

[41] E. O. Brigham, "The Fast Fourier Transform (FFT)", in The Fast Fourier Transform and Its Applications. Prentice Hall, 1988, pp. 131166.

[42] LabVIEW, NI. [Online]. Available: https://www.ni.com/enrs/shop/labview.html

[43] M. Dimitrijević, M. Andrejević-Stošović, and D. Stevanović, "Nonlinear loads classification method using alternative reactive power definitions", in Proc. of 14th International Conference on Applied Electromagnetics, Niš, Serbia, 2019, pp. 1-4.

[44] O. Nieto-Taladriz, M. Dimitrijević, D. Stevanović, and D. Mirković, "Energy profile of a personal computer", in Proc. of Zbornik LVI konferencije ETRAN, Zlatibor, 2012.

This article is an open access article distributed under the terms and conditions of the Creative Commons Attribution 4.0 (CC BY 4.0) license (http://creativecommons.org/licenses/by/4.0/). 